\title{
LYAPUNOV'S FUNCTIONS AND EXISTENCE OF INTEGRAL MANIFOLDS FOR IMPULSIVE DIFFERENTAL SYSTEMS WITH TIME-VARYING DELAY*
}

\author{
GANI STAMOV ${ }^{\dagger}$
}

\begin{abstract}
In this paper the existence of integral manifolds for impulsive differential systems with time-varying delay and with impulsive effect at fixed moments are investigated. The main results are obtained by using of piecewise continuous Lyapunov's functions and Razumikhin's technique.
\end{abstract}

Key words. Integral manifold, impulsive differential systems.

AMS subject classifications. 34A37, 42B25

1. Introduction. The theory of integral manifolds comes back from the works of A. M. Lyapunov (see [1]) for qualitative theory of differential equations, and N. N. Bogolubov, U. A. Mitropolskii (see [14]) for asymptotic methods of nonlinear mechanics.

In recent years the theory of integral manifolds for impulsive differential equations have been intensively researched (see [2-4], [8]). Recently, some properties (existence, reduction of the systems, asymptotic stability) are investigated by several authors (see [6-7], [9], [12-13], [16]). A natural generalization of impulsive ordinary differential equations is impulsive differential equations with time-varying delay. In spite of great possibilities for applications, the theory of these equations is developing rather slowly due to a series of difficulties of technical and theoretical character yet.

In this paper we apply the comparison principle to the problem of existence of integral manifolds for impulsive differential equations with time-varying delay. The impulses are in the fixed moments of time and since the solutions of such systems are piecewise continuous functions. The investigations are carried out by using minimal subset of a suitable space of piecewise continuous functions, by the elements of which the derivative of Lyapunov's functions are estimated [13], [18].

2. Preliminary notes. Let $\mathbb{R}^{n}$ be the $n$-dimensional Euclidean space with norm $\|\|,. \mathbb{R}^{+}=[0, \infty), B_{h}=\left\{x \in \mathbb{R}^{n}:\|x\| \leq h\right\}, h>0, \Omega \subset \mathbb{R}^{n}, B_{h} \subset \Omega$.

Consider the sets:

$B=\left\{\left\{\tau_{k}\right\} ; k \in \mathbb{Z}, \tau_{k} \in \mathbb{R}, \tau_{k}<\tau_{k+1}, \lim _{k \rightarrow+\infty} \tau_{k}=+\infty\right\}$ is the set of all sequences unbounded and strictly increasing.

$P C\left[\mathbb{R}, \mathbb{R}^{n}\right]=\left\{\varphi: \mathbb{R} \rightarrow \mathbb{R}^{n}, \varphi\right.$ is a piecewise continuous function with points of discontinuity at the first kind $\tau_{k},\left\{\tau_{k}\right\} \in B$ at which $\varphi\left(\tau_{k}^{-}\right)$and $\varphi\left(\tau_{k}^{+}\right)$exist, and $\left.\varphi\left(\tau_{k}^{-}\right)=\varphi\left(\tau_{k}\right)\right\}$.

$P C^{1}\left[\mathbb{R}, \mathbb{R}^{n}\right]=\left\{\varphi: \mathbb{R} \rightarrow \mathbb{R}^{n}, \varphi\right.$ is continuously differentiable everywhere except the poins $\tau_{k},\left\{\tau_{k}\right\} \in B$ at which $\dot{\varphi}\left(\tau_{k}^{-}\right)$and $\dot{\varphi}\left(t_{k}^{+}\right)$exist, and $\left.\dot{\varphi}\left(\tau_{k}^{-}\right)=\dot{\varphi}\left(\tau_{k}\right)\right\}$.

Let $\varphi_{0} \in P C[\mathbb{R}, \Omega]$ and $\left|\varphi_{0}\right|=\sup _{t \in \mathbb{R}}\left\|\varphi_{0}(t)\right\|$. Consider the following system of impulsive differential equations with time-varying delay

$$
\left\{\begin{array}{l}
\dot{x}(t)=f(t, x(t), x(t-\eta(t))), t \geq t_{0}, t \neq \tau_{k}, \\
x(t)=\varphi_{0}(t), t \in\left(-\infty, t_{0}\right], \\
\Delta x\left(\tau_{k}\right)=x\left(\tau_{k}^{+}\right)-x\left(\tau_{k}\right)=I_{k}\left(x\left(\tau_{k}\right)\right), \tau_{k} \geq t_{0}, k=1,2, \ldots,
\end{array}\right.
$$

${ }^{*}$ Received June 16, 2008; accepted for publication June 26, 2009.

$\dagger$ Department of Mathematics, Technical University - Sofia, Sliven, Bulgaria (gstamov@abv.bg). 
where:

i) $f:\left(t_{0}, \infty\right) \times \Omega \times \Omega \rightarrow \mathbb{R}^{n}, t \in \mathbb{R}, \eta: \mathbb{R} \rightarrow \mathbb{R}^{+}$;

ii) $I_{k}: \Omega \rightarrow \mathbb{R}^{n}$;

iii) $\left\{\tau_{k}\right\} \in B, 0 \leq t_{0} \equiv \tau_{0} \leq \tau_{1}<\tau_{2}<\ldots$;

iv) $\varphi_{0}(t) \in \Omega, t_{0} \in\left(-\infty, t_{0}\right]$.

Denote by $x(t)=x\left(t ; t_{0}, \varphi_{0}\right)$ the solution of system (1) and by $J^{+}\left(t_{0}, \varphi_{0}\right)$ - the maximal interval of type $\left[t_{0}, \beta\right)$ in which the solution $x\left(t ; t_{0}, \varphi_{0}\right)$ is defined, and by $\theta_{+}\left(t_{0}, \varphi_{0}(t)\right)$ denote the integral orbit of the solution $x\left(t ; t_{0}, \varphi_{0}\right)$ for $t \in J^{+}\left(t_{0}, \varphi_{0}\right)$.

We assume that the solution $x(t)=x\left(t ; t_{0}, \varphi_{0}\right)$ of (1) exists and from [13] is easy to see that $x(t)$ is piecewise continuous function with points of discontinuity at the moments $\tau_{k}, k=1,2, .$. at which it is continuous from the left.

Thus in interval $J^{+}\left(t_{0}, \varphi_{0}\right)$ the solution $x\left(t ; t_{0}, \varphi_{0}\right)$ of problem (1) is a piecewise continuous function with points of discontinuity of the first kind $\tau_{k}, k=1,2, \ldots$ at which it is continuous from the left.

Further on the paper we are going to write $x>0$ if $x_{i}>0, i=1,2, \ldots, n$ or $x \leq 0$ if $x_{i} \leq 0, i=1,2, \ldots, n$.

Introduce the following assumptions:

H1. The function $f \in C\left[\left[t_{0}, \infty\right) \times \Omega \times \Omega, R^{n}\right]$ and $f$ is Lipschitz continous with respect to its second and third arguments in $\left[t_{0}, \infty\right) \times \Omega \times \Omega$.

H2. $\eta \in C\left[\mathbb{R}, \mathbb{R}^{+}\right], t-\eta(t) \geq 0$.

H3. There exists $N=$ const $>0$ such that

$$
|f(t, x, \bar{x})| \leq N<\infty,(t, x, \bar{x}) \in\left[t_{0}, \infty\right) \times \Omega \times \Omega .
$$

H4. The function $I_{k} \in C\left[\Omega, R^{n}\right]$ and $I_{k}$ are Lipschitz continous in $\Omega$ for $k=1,2, \ldots$

H5. The functions $\left(I+I_{k}\right): \Omega \rightarrow \Omega, k=1,2, \ldots$, where $I$ is the identity in $\Omega$.

H6. $\varphi_{0} \in P C[\mathbb{R}, \Omega]$.

\section{Lemma 1. Let the conditions H1-H6 hold.}

Then $J^{+}\left(t_{0}, \varphi_{0}\right)=\left[t_{0}, \infty\right)$.

Proof. Since the conditions H1-H3 hold then from the existence theorem for the functional differential equation without impulses [10] it follows that the solution $x(t)=x\left(t ; t_{0}, \varphi_{0}\right)$ of the problem (1) is defined on each of the intervals $\left(\tau_{k-1}, \tau_{k}\right]$, $k=1,2, \ldots, t_{0}=\tau_{0}$. From H4-H6 and the property of the sequence $\left\{\tau_{k}\right\}, k=1,2, \ldots$ we conclude that it is continually for $t \geq t_{0}$.

Definition 1 . We call an arbitrary manifold $M$ in the extended phase space $\mathbb{R} \times \Omega$ of (1) an integral manifold if from $\left(t, \varphi_{0}(t)\right) \in M$ for $t \leq t_{0}$ it follows that, 
$\theta_{+}\left(t_{0}, \varphi_{0}(t)\right) \subset M$

Consider the following sets and classes of functions:

$$
\begin{aligned}
& G_{k}=\left\{(t, x, y) \in \mathbb{R} \times \mathbb{R}^{n} \times \mathbb{R}^{n}: \tau_{k-1}<t<\tau_{k}, k=1,2, \ldots\right\}, \\
& G=\bigcup G_{k}, k=1,2, \ldots, \\
& \quad V_{M}=\left\{V \in C\left[G, \mathbb{R}^{+}\right], V(t, x)=0 \text { for }(t, x) \in M, t \geq t_{0},\right. \\
& \text { and } V(t, x)>0 \text { for }(t, x) \in\left[t_{0}, \infty\right) \times \Omega \backslash M, V \text { is locally Lipschitz } \\
& \text { continuous with respect to its second argument } x \text { in each of the } \\
& \text { sets } G_{k}, V\left(\tau_{k}^{-}, x\right)=V\left(\tau_{k}, x\right), \text { and } V\left(\tau_{k}^{+}, x\right)=\lim _{t \rightarrow \tau_{k}} V(t, x) \\
& \text { exists, } k=1,2, \ldots\} . \\
& \Omega_{1}=\left\{x \in P C[\mathbb{R}, \Omega]: V(s, x(s)) \leq V(t, x(t)), s \in(-\infty, t], t \geq t_{0}, V \in V_{M}\right\} .
\end{aligned}
$$

We note that manifold $M$ is a kernel of the class $V_{M}$ and let $V \in V_{M}$. Introduce

$$
D^{+} V(t, x(t))=\lim _{\sigma \rightarrow 0^{+}} i n f \sigma^{-1}[V(t+\sigma, x(t)+\sigma f(t, x(t), x(t-\eta(t))))-V(t, x(t))] .
$$

LEMma 2. Let the following conditions hold:

1. Conditions $\mathbf{H 1}, \mathbf{H 2}, \mathbf{H} 4$ and $\mathbf{H 5}, \mathbf{H} 6$ are met.

2. The function $g:\left(t_{0}, \infty\right) \times \mathbb{R}^{+} \rightarrow \mathbb{R}^{+}$is continuous in each of the sets $\left(\tau_{k-1}, \tau_{k}\right] \times \mathbb{R}^{+}, \quad k=1,2, \ldots$ and $g(t, 0)=0$ for $t \in\left(t_{0}, \infty\right)$.

3. $B_{k} \in C\left[\mathbb{R}^{+}, \mathbb{R}^{+}\right], B_{k}(0)=0$ and $\psi_{k}(u)=u+B_{k}(u), k=1,2, \ldots$ are nondecreasing with respect to $u$.

4. The maximal solution $r\left(t ; t_{0}, u_{0}\right)$ of the problem

$$
\left\{\begin{array}{l}
\dot{u}=g(t, u), t>t_{0}, t \neq \tau_{k}, \\
u\left(t_{0}^{+}\right)=u_{0} \geq 0 \\
\Delta u\left(\tau_{k}\right)=B_{k}\left(u\left(\tau_{k}\right)\right), \tau_{k} \geq t_{0}, k=1,2, \ldots
\end{array}\right.
$$

is defined in the interval $\left[t_{0}, \infty\right)$.

5. The solution $x=x\left(t ; t_{0}, \varphi_{0}\right)$ of the problem (1) is such that $x \in$ $P C[\mathbb{R}, \Omega] \cap P C^{1}\left[\left[t_{0}, \infty\right), \Omega\right]$. 
6. There exists a function $V \in V_{M}$ is such that $V\left(t_{0}^{+}, \varphi_{0}(0)\right) \leq u_{0}$ and the following relations are satisfied

$$
\begin{gathered}
D^{+} V(t, x(t)) \leq g(t, V(t, x(t))), t \neq \tau_{k}, k=1,2, \ldots, t>t_{0}, \\
V(s, x(s)) \leq V(t, x(t)) \text { for any } s \leq t
\end{gathered}
$$

and

$$
V\left(\tau_{k}^{+}, x\left(\tau_{k}\right)+I_{k}\left(x\left(\tau_{k}\right)\right)\right) \leq \psi_{k}\left(V\left(\tau_{k}, x\left(\tau_{k}\right)\right)\right), k=1,2, \ldots
$$

Then

$$
V\left(t, x\left(t ; t_{0}, \varphi_{0}\right)\right) \leq r\left(t ; t_{0}, u_{0}\right), t \in\left[t_{0}, \infty\right)
$$

Proof. From Lemma 1 it follows that $J^{+}\left(t_{0}, \varphi_{0}\right)=\left[t_{0}, \infty\right)$.

The maximal solution $r\left(t ; t_{0}, u_{0}\right)$ of the problem (2) is defined by the equality

$$
r\left(t ; t_{0}, u_{0}\right)=\left\{\begin{array}{l}
r\left(t ; \tau_{0}, u_{0}^{+}\right), \tau_{0}<t \leq \tau_{1}, \\
r_{1}\left(t ; \tau_{1}, u_{1}^{+}\right), \tau_{1}<t \leq \tau_{2}, \\
\ldots \ldots \ldots \ldots \ldots \ldots \ldots \ldots \ldots \ldots \ldots . \\
r_{k}\left(t ; \tau_{k}, u_{k}^{+}\right), \tau_{k}<t \leq \tau_{k+1}, \\
\ldots \ldots \ldots \ldots \ldots \ldots \ldots \ldots \ldots \ldots . . .
\end{array}\right.
$$

where $r_{k}\left(t ; \tau_{k}, u_{k}^{+}\right)$is the maximal solution of the equation without impulses $\dot{u}=g(t, u)$ in the interval $\left(\tau_{k}, \tau_{k+1}\right], k=0,1,2, \ldots$, for which $u_{k}^{+}=$ $\psi_{k}\left(r_{k-1}\left(\tau_{k} ; \tau_{k-1}, u_{k-1}^{+}\right)\right), k=1,2, \ldots$ and $u_{0}^{+}=u_{0}$.

Let $t \in\left(t_{0}, \tau_{1}\right]$. Then from the continuous case ([10], Theorem 1.4.1,) it follows that

$$
V\left(t, x\left(t ; t_{0}, \varphi_{0}\right)\right) \leq r\left(t ; t_{0}, u_{0}\right),
$$

i.e., the inequality $(3)$ is valid for $t \in\left(\tau_{0}, \tau_{1}\right]$.

Suppose that (3) is satisfied for $t \in\left(\tau_{k-1}, \tau_{k}\right], k>1$. Then, using condition 6 of Lemma 2 and the fact that the function $\psi_{k}$ is nondecreasing, we obtain

$$
\begin{gathered}
V\left(\tau_{k}^{+}, x\left(\tau_{k}^{+} ; t_{0}, \varphi_{0}\right)\right) \leq \psi_{k}\left(V\left(\tau_{k}, x\left(\tau_{k} ; t_{0}, \varphi_{0}\right)\right)\right) \leq \psi_{k}\left(r\left(\tau_{k} ; t_{0}, \varphi_{0}\right)\right) \\
=\psi_{k}\left(r_{k-1}\left(\tau_{k} ; \tau_{k-1}, u_{k-1}^{+}\right)\right)=u_{k}^{+}
\end{gathered}
$$

We apply again Theorem 1.4.1 from [10] for the continuous case in the interval $\left(\tau_{k}, \tau_{k+1}\right]$ and obtain

$$
V\left(t, x\left(t ; t_{0}, \varphi_{0}\right)\right) \leq r_{k}\left(t ; \tau_{k}, u_{k}^{+}\right)=r\left(t ; t_{0}, u_{0}\right),
$$

i. e., the inequality (3) is valid for $t \in\left(\tau_{k}, \tau_{k+1}\right]$.

The proof is completed by induction. 
In the case when $g(t, u)=0$ for $(t, u) \in\left(t_{0}, \infty\right) \times \mathbb{R}^{+}$and $\psi_{k}(u)=u$ for $u \in \mathbb{R}^{+}, \quad k=1,2, \ldots$ we deduce the following corollary from Lemma 2 .

Corollary 1. Let the following conditions hold:

1. Conditions $\mathbf{H 1}, \mathbf{H 2}, \mathbf{H} 4$ and $\mathbf{H 5}, \mathbf{H} 6$ are met.

2. The condition 5 of Lemma 2 is satisfied.

3. The function $V \in V_{M}$ is such that the inequalities

$$
\begin{gathered}
D_{-} V(t, x(t)) \leq 0, t \neq \tau_{k}, k=1,2, \ldots, \\
V\left(t^{+}, x(t)+I_{k}(x(t))\right) \leq V(t, x(t)), k=1,2, \ldots
\end{gathered}
$$

are valid for $t \in\left[t_{0}, \infty\right)$ and $x \in \Omega_{1}$.

Then

$$
V\left(t, x\left(t ; t_{0}, \varphi_{0}\right)\right) \leq V\left(t_{0}^{+}, \varphi_{0}\left(t_{0}\right)\right), t \in\left[t_{0}, \infty\right)
$$

\section{Main results.}

Theorem 1. Assume that:

1. Conditions H1-H6 are met.

2. For the problem (1) there exists a function $V \in V_{M}$ with kernel the manifold $M$, so that the following relations are satisfied

$$
\begin{aligned}
& D_{-} V(t, x(t)) \leq 0, t \neq \tau_{k}, k=1,2, \ldots, t \geq t_{0}, x \in \Omega_{1}, \\
& V\left(\tau_{k}^{+}, x\left(\tau_{k}\right)+I_{k}\left(x\left(\tau_{k}\right)\right)\right) \leq V\left(\tau_{k}, x\left(\tau_{k}\right)\right), \quad k=1,2, \ldots .
\end{aligned}
$$

Then $M$ is an integral manifold for (1).

Proof. Suppose that $M$ is not an integral manifold. Therefore there exists $t^{\prime}, t^{\prime}>$ $t_{0}$ such that, if $\left(t, \varphi_{0}(t)\right) \in M$ for $t \in\left[-\infty, t_{0}\right]\left(t, x\left(t ; t_{0}, \varphi_{0}\right)\right) \in M$ for $t_{0}<t \leq t^{\prime}$ and $\left(t, x\left(t ; t_{0}, \varphi_{0}\right)\right) \notin M$ for $t>t^{\prime}$.

Then $V\left(t^{\prime}, x^{\prime}\right)=0$, where $x^{\prime}=x\left(t^{\prime} ; t_{0}, \varphi_{0}\right)$. Moreover $x(t) \in P C\left[J^{+}\left(t_{0}, \varphi_{0}\right), \mathbb{R}^{n}\right]$.

We denote that for $t^{\prime}$ the following two cases are possible:

a) If $t^{\prime}=\tau_{k}, k=j, j+1, \ldots, j \geq 1$, then $\left(t^{\prime+}, x\left(t^{\prime+} ; t_{0}, \varphi_{0}\right)\right)=\left(t^{\prime+}, x\left(t^{\prime} ; t_{0}, \varphi_{0}\right)+\right.$ $\left.I_{k}\left(x^{\prime}\right)\right),\left(t^{\prime+}, x\left(t^{\prime+} ; t_{0}, \varphi_{0}\right)\right) \notin M$ and consequently $V\left(t^{\prime+}, x\left(t^{\prime+} ; t_{0}, \varphi_{0}\right)\right)>0$. Then $0=V\left(t^{\prime}, x^{\prime}\right)<V\left(t^{\prime+}, x\left(t^{\prime+} ; t_{0}, \varphi_{0}\right)\right)$ which is contradiction by $(5)$.

b) If $t^{\prime} \neq \tau_{k}, k=j, j+1, \ldots, j \geq 1$ there exists $t^{\prime \prime}>t^{\prime}$ such that $\left(t^{\prime \prime}, x\left(t^{\prime \prime} ; t_{0}, \varphi_{0}\right)\right) \notin$ $M$. From (4) and (5) it follows that the function $V(t, x(t))$ is not increasing in $\left[t_{0}, \infty\right)$ and

$$
V\left(t^{\prime \prime}, x\left(t^{\prime \prime} ; t_{0}, \varphi_{0}\right)\right)>0 \text {. }
$$


Since the conditions of Corollary 1 are met, then

$$
V\left(t, x\left(t ; t_{0}, \varphi_{0}\right)\right) \leq V\left(t^{\prime}, x\left(t^{\prime} ; t_{0}, \varphi_{0}\right)\right),
$$

for $t \in\left[t^{\prime}, \infty\right)$ and we obtain

$$
V\left(t^{\prime \prime}, x\left(t^{\prime \prime} ; t_{0}, \varphi_{0}\right)\right) \leq V\left(t^{\prime}, x\left(t^{\prime} ; t_{0}, \varphi_{0}\right)\right)=0,
$$

which is contradicts (6).

The proof of Theorem 1 is complete.

Now we shall use Theorem 1 to prove the existence of integral manifold for the system

$$
\left\{\begin{array}{l}
\dot{x}(t)=\left\{\begin{array}{l}
A(t) x(t)+B(t) x(t-\eta(t)), x(t)>0, t \neq \tau_{k} \\
0, \quad x(t) \leq 0, t \neq \tau_{k}
\end{array}\right. \\
\Delta x(t)=\left\{\begin{array}{l}
C_{k} x(t), x(t)>0, t=\tau_{k} \\
0, \quad x(t) \leq 0, t=\tau_{k}
\end{array}\right.
\end{array}\right.
$$

where:

i) $t>t_{0} ; x \in P C\left[\left(t_{0}, \infty\right), \mathbb{R}^{n}\right] ; A(t)$ and $B(t)$ are $(n \times n)$ matrix- valued functions;

ii) $C_{k}, k=1,2, \ldots$ are $(n \times n)$ matrices; $\eta \in C\left[\left(t_{0}, \infty\right), \mathbb{R}^{+}\right]$;

iii) $\left\{\tau_{k}\right\} \in B, 0 \leq t_{0} \equiv \tau_{0} \leq \tau_{1}<\tau_{2}<\ldots$

Let $\tau=\inf _{t \geq t_{0}}(t-\eta(t))$ and $\varphi_{1} \in C\left[\left[\tau, t_{0}\right], \mathbb{R}^{n}\right]$. Denote by $x(t)=x\left(t ; t_{0}, \varphi_{1}\right)$ the solution of the system (7) satisfying the initial condition :

$$
x\left(t ; t_{0}, \varphi_{1}\right)=\varphi_{1}(t), \tau \leq t \leq t_{0},
$$

and by $J^{+}\left(t_{0}, \varphi_{1}\right)$ - the maximal interval of the type $\left(t_{0}, \beta\right)$, at which the solution $x\left(t ; t_{0}, \varphi_{1}\right)$ is defined.

Theorem 2. Assume that:

1. The matrix functions $A(t)$ and $B(t)$ are continuous for $t \in\left(t_{0}, \infty\right)$.

2. The condition $\mathbf{H 2}$ is met.

3. For each $k=1,2, \ldots$ the elements of the matrix $C_{k}$ are nonnegative.

4. There exists a continuous real $(n \times n)$ matrix $D(t), t \in\left(t_{0}, \infty\right)$, which is symmetric, positively definite, differentiable for $t \neq \tau_{k}, k=1,2, \ldots$ and such that for each $k=1,2, \ldots$

$$
\begin{gathered}
x^{T}\left[A^{T}(t) D(t)+D(t) A(t)+\dot{D}(t)\right] x \leq-c(t)|x|^{2}, x \in \mathbb{R}^{n}, t \neq \tau_{k}, \\
x^{T}\left[C_{k}^{T} D(t)+D(t) C_{k}+C_{k}^{T} D(t) C_{k}\right] x \leq 0, t=\tau_{k},
\end{gathered}
$$


where $c(t)>0$ is a continuous function.

5. There exists an integral positive function $\lambda(t)$ such that

$$
\begin{aligned}
d(t)= & c(t)-\max \{\alpha(t) \lambda(t), \beta(t) \lambda(t)\} \geq 0, \\
& \frac{2 \beta^{1 / 2}(t)}{\alpha^{1 / 2}(t-\eta(t))}|D(t) B(t)| \leq d(t),
\end{aligned}
$$

where $\alpha(t)$ and $\beta(t)$ are respectively the smallest and the greatest eingenvalues of matrix $D(t)$.

Then, the set $M=\left\{\left[\tau-t_{0}, \infty\right) \times\left\{x \in \mathbb{R}^{n}: x \leq 0\right\}\right\}$ is integral manifold of the system (7).

Proof. Consider the function

$$
V(t, x)=\left\{\begin{array}{l}
x^{T} D(t) x, \text { for } x>0 \\
0, \text { for } x \leq 0
\end{array}\right.
$$

From condition 5 of Theorem 2 the following inequalities hold

$$
\alpha(t)|x|^{2} \leq x^{T} D(t) x \leq \beta(t)|x|^{2} .
$$

For the function $V(t, x)$ the set $\Omega_{1}$ is

$$
\begin{aligned}
& \Omega_{1}=\left\{x \in P C\left[\left(t_{0}, \infty\right), \mathbb{R}^{n}\right]: x^{T}(s) D(s) x(s) \leq x^{T}(t) D(t) x(t), t-\eta(t) \leq s \leq t,\right. \\
& \left.\quad t \in\left(t_{0}, \infty\right)\right\} .
\end{aligned}
$$

If $t>t_{0}$ and $x \in \Omega_{1}$ we have

$$
\begin{aligned}
\alpha(t-\eta(t))|x(t-\eta(t))|^{2} & \leq x^{T}(t-\eta(t)) D(t-\eta(t)) x(t-\eta(t)) \\
& \leq x^{T}(t) D(t) x(t) \leq \beta(t)|x(t)|^{2},
\end{aligned}
$$

and we obtain the estimate

$$
|x(t-\eta(t))| \leq \frac{\beta^{1 / 2}(t)}{\alpha^{1 / 2}(t-\eta(t))}|x(t)| .
$$

Let $t \neq \tau_{k}$ and $x \in \Omega_{1}$. From (9), (11), (12) and (13) we have

$$
\begin{aligned}
D_{-} V(t, x(t)) & =\left\{\begin{array}{l}
-c(t)|x(t)|^{2}+2|D(t) B(t)||x(t)||x(t-h)|, x(t)>0, \\
0, \quad x(t) \leq 0
\end{array}\right. \\
& \leq \begin{cases}-[c(t)-d(t)]|x(t)|^{2}, x(t)>0, \\
0, & x(t) \leq 0\end{cases} \\
& \leq-\lambda(t) V(t, x(t)) .
\end{aligned}
$$

Let $t=\tau_{k}$. Then from (10) we get

$$
\begin{aligned}
& V\left(t^{+}, x(t)+C_{k} x(t)\right) \\
= & \begin{cases}\left(x^{T}(t)+x^{T}(t) C_{k}^{T}\right) D(t)\left(x(t)+C_{k} x(t)\right), x(t)>0, \\
0, & x(t) \leq 0\end{cases} \\
= & \begin{cases}x^{T}(t) D(t) x(t)+x^{T}(t)\left[C_{k}^{T} D(t)+D(t) C_{k}+C_{k}^{T} D(t) C_{k}\right], x(t)>0, \\
0, & x(t) \leq 0\end{cases} \\
\leq & V(t, x(t)) .
\end{aligned}
$$


Thus we have checked that all the conditions of Theorem 1 are satisfied.

Hence the set $M=\left\{\left[\tau-t_{0}, \infty\right) \times\left\{x \in \mathbb{R}^{n}: x \leq 0\right\}\right\}$ is an integral manifold for the system (7).

\section{REFERENCES}

[1] A. M. Lyapunov, The Main Problems of Stability of the Motions, Nauka, Moscow, 1950. (in Russian)

[2] A. M. Samoilenko and N. A. Perestyuk, Differential Equations with Impulse Effect, World Scientific, Singapore, 1995.

[3] G. Stamov, Integral Manifolds of Singularly Perturbed Systems of Impulsive Differential Equations Defined on Tori, Ann. Univ. Ferrara - Sez. VII - Sc. Mat., XLI (1995), pp. 117-130.

[4] G. Tr. Stamov, Stability of Moving Invariant Conditionally Manifolds for Impulsive Differential Equations, Adv. Studies in Cont. Math, 9/1 (2004), pp. 7-14.

[5] D. D. Bainov and I. M. Stamova, Second Method of Lyapunov and Comparison Principle for Impulsive Differential-Difference Equations, J. Austra. Math. Soc. Ser. B, 38 (1997), pp. 489-505.

[6] D. D. Bainov and I. M. Stamova, Strong Stability of Impulsive Differential-Difference Equations, PanAmerican Math.J., 9 (1999), pp. 87-95.

[7] D. D. Bainov and I. M. Stamova, Lypschitz Stability of Impulsive Functional Differential Equations, ANZIAM J., 42 (2001), pp. 504-515.

[8] D. Bainov, S. Kostadinov and NguYen Van Min, Dichotomies and Integral Manifolds of Impulsive Differential Equations, SCT Publishing, Singapore, 1994.

[9] D. Bainov and P. Simeonov, Systems with Impulse Effect: Stability, Theory and Applications, Ellis Horwood, Chichester, 1989.

[10] J. K. Hale, Theory of Functional Differential Equations, Springer-Verlag, New York, Heidelberg, Berlin, 1977.

[11] J. K. Hale And V. Lunel, Introduction to Functional Differential Equations, Springer-Verlag, 1993.

[12] I. M. Stamova, Stability Theorems of Perturbed Linear Impulsive Equations, Math. Science Research J., 2 (2002), pp. 96-103.

[13] I. M. Stamova AND G. T. Stamov, Lyapunov-Razumikhin Method for Impulsive Functional Differential Equations and Applications to the Population Dynamics, J. Comp.Apll. Math., 130 (2001), pp. 163-171.

[14] N. N. Bogolubov and U. A. Mitropolskir, Asymptoticall Methods in Nonlinear Mechanics, Nauka, Moscow, 1974. (in Russian)

[15] V. B. Kolmanovskit And V. R. Nosov, Stability of Functional Differential Equations, Academic Press, 1986.

[16] V. Lakshmikantham, D. D. Bainov and P. S. Simeonov, Theory of Impulsive Differential Equations, World Scientific, Singapore, 1989.

[17] Z. Luo And J. Shen, Stability Results for Impulsive Functional Differential Equations with Infinite Delays, J. Comp. Apll. Math., 131 (2001), pp. 55-64.

[18] B. S. Razumikhin, Stability of Systems with Retardation, Nauka, Moscow, 1988. (in Russian) 